\title{
Aprendizaje Basado en Proyectos Dentro de un Curso Universitario de Interacción Humano Computadora
}

Learning Based on Projects Within a University Course of Human Computer Interaction

Guadalupe Toledo Toledo'

gtoledo@sandunga.unistmo.edu.mx

J. Jesús Arellano Pimentel ${ }^{1}$

jjap@sandunga.unistmo.edu.mx

Francisco Aguilar Acevedo'

aguilar.afco@sandunga.unistmo.edu.mx

Edwin Williams Molina Rodríguez'

williamsrdz495@gmail.com

Universidad del Istmo, Departamento de Ingeniería en Computación, México. 
Resumen: Hablar de un software de calidad involucra tratar con dos disciplinas importantes, la ingeniería de software y la interacción humano computadora, esta segunda atiende el desarrollo bajo el acompañamiento de los usuarios finales, por lo que resulta atractivo integrar el aprendizaje basado en proyectos como seguimiento de un curso universitario, los cuales estarán diseñados a corto plazo resolviendo una problemática real y afín al estudiante con la intensión que éste se comprometa en proporcionar su solución, tomando como guía curricular el diseño centrado en el usuario. La determinación de los puntos de control bajo esta metodología ha permitido relacionar con éxito los conceptos de la asignatura con proyectos que ofrezcan un beneficio a la sociedad, al cierre de las actividades y productos solicitados se espera que los estudiantes puedan desarrollar artículos de divulgación.

Palabras clave: Aprendizaje basado en proyectos, Interacción humano computadora, Diseño centrado en el usuario, Pruebas de usabilidad.

Abstract: Talking about quality software involves dealing with two important disciplines, software engineering and human computer interaction, this second deals with development under the guidance of end users, so it is attractive to integrate project-based learning as a follow-up to a university course, which will be designed in the short term solving a real problem and related to the student with the intention that this is committed to provide your solution, taking as a curriculum guide the user-centered design. The determination of the control points under this methodology has made it possible to successfully relate the concepts of the subject with projects that offer a benefit to society. At the closing of the activities and products requested, it is expected that students can develop dissemination articles.

Keywords: Project-based learning, human computer interaction, user-centered design, usability testing. 


\section{Introducción}

La influencia que ha comenzado a adquirir la Interacción Humano Computadora (IHC) junto al de usabilidad para el desarrollo de aplicaciones efectivas, eficientes y que satisfacen el gusto de los usuarios, compactando con ello el concepto de calidad, ha abierto a debate la finalidad que le corresponde a la ingeniería de software como el marco conceptual más influyente en la construcción de aplicaciones que automatizan procesos y que a su vez son considerados de calidad.

Y es que, existe una diferencia trascendental entre estos dos enfoques respecto a la calidad del producto de software, por una parte la Ingeniería de Software según (S. Pressman, 2006) se encarga de cumplir requerimientos funcionales solicitados por un cliente, empleando las mejores prácticas para dar seguimiento al desarrollo y garantizar la ausencia de errores. No obstante, los clientes que son tratados en este formalismo no son necesariamente los mismos que utilizarán el sistema, sino son solo quienes indicarán sus necesidades y pagarán por el producto, para posteriormente implantar el sistema en su modelo de negocios e invertir en el proceso de capacitación que permita su aceptación por parte de sus empleados.

Sin embargo, la calidad en IHC radica en la idea de que el software debe estar definido bajo la participación integral del usuario final (persona quien realmente utilizará el sistema) en la construcción y determinación de cada fase del software (AENOR, 2000). Ya que, el criterio más importante para la aceptación del producto final reside en lograr interpretar el modelo mental de estos usuarios al realizar sus procesos en cotidianeidad para posteriormente replicarlos en el software, logrando así su aceptación de manera inmediata, con muy poca o nula necesidad de capacitación.

Por tanto, la integración de ambas disciplinas es de relevancia para obtener productos de calidad, es decir, desde la aceptación del cliente con el que trata el equipo de desarrollo (persona que financia el producto y quién da los requerimientos) como la aceptación del usuario final. Por lo que la transmisión de ese conocimiento y su aclaración es de vital importancia durante la formación de profesionistas en el área de cómputo (quiénes se convertirán en desarrolladores de software).

Bajo esta consigna e independientemente del orden en que son atendidos estos tópicos dentro del marco curricular de las carreras universitarias afines, y debido a que la ingeniería de software es un rubro mejor documentado para su comprensión, se busca partir de atender la participación de la IHC en la integración de conceptos preliminares para la construcción del software, el cual se hace bajo la metodología del Diseño Centrado en el Usuario (DCU) (AENOR, 
2000). Esta metodología atiende los factores relacionados con la usabilidad del software mediante la norma ISO 13407, así como la identificación de artefactos o herramientas para cubrir sus cuatro etapas.

Una estrategia útil para la incursión de estos conceptos dentro de un curso universitario se da bajo el esquema del Aprendizaje basado en proyectos (ABP) (Coria Arreola, 2011), ya que resulta trascendental involucrar a los estudiantes bajo un escenario real que les permita asociar su nuevo conocimiento con una experiencia afín a sus intereses así como también contribuir a la sociedad y/o escuela con soluciones de software de calidad.

El presente trabajo es una propuesta metodológica para el desarrollo de un curso de IHC, llevado a cabo en la Universidad del Istmo, ubicado en Santo Domingo Tehuantepec, en el estado de Oaxaca; tomando como eje principal el DCU y la estrategia ABP. Se espera que el estudiante adquiera los conocimientos elementales del enfoque centrado en el usuario para el desarrollo de software de calidad y a su vez desarrolle capacidades de emprendimiento para proponer soluciones que representen beneficios a la sociedad, logrando con ello obtener mejores competencias para su vida profesional.

\section{Trabajo Relacionado}

Incorporar a un curso de IHC los conceptos del DCU, implica consultar la norma ISO 13407 (AENOR, 2000). EI DCU busca entender cuáles son las necesidades del usuario, lo que sienten y cómo piensan, usando herramientas específicas para poder obtener esta información en cada una de las etapas arriba señaladas. Sin embargo, la decisión de esas herramientas y actividades no son aspectos triviales que puedan encontrarse descritas fácilmente en el estado del arte y mucho menos desglosadas para un curso Universitario.

Se han encontrado cursos de especialización sobre IHC en instituciones de educación superior (véase Tabla 1). Sin embargo, la integración de la Metodología del DCU es evidente en algunas de ellas, y pese a eso resulta difícil identificar herramientas y/o mecanismos para cumplir con sus etapas, así como tampoco se logró identificar la estrategia de aprendizaje empleada, es decir, si estuviesen empleando ABP, éste no se describe claramente en sus programas del curso. 


\begin{tabular}{|c|c|c|c|c|}
\hline Institución & Asignatura & Duración & Temas Principales & $\begin{array}{l}\text { Nivel } \\
\text { académico }\end{array}$ \\
\hline $\begin{array}{l}\text { (Universidad de } \\
\text { Chile, 2017) }\end{array}$ & $\begin{array}{l}\text { Curso de } \\
\text { experiencias de } \\
\text { usuario en sitios } \\
\text { web y soportes } \\
\text { digitales }\end{array}$ & $\begin{array}{l}28 \text { hrst } \\
\text { en } 7 \\
\text { sesiones }\end{array}$ & $\begin{array}{l}\text { Metodología DCU, estrategias } \\
\text { de recolección de requisitos, } \\
\text { pruebas de usabilidad }\end{array}$ & Licenciatura \\
\hline $\begin{array}{l}\text { (Universidad } \\
\text { Autónomar de } \\
\text { Barcelona, 2017) }\end{array}$ & $\begin{array}{l}\text { Curso de Diseño } \\
\text { Centrado en el } \\
\text { usuario }\end{array}$ & $\begin{array}{l}\text { No lo } \\
\text { especifica }\end{array}$ & $\begin{array}{l}\text { Metodología DCU, estrategias } \\
\text { de recolección de requisitos, } \\
\text { pruebas de usabilidad }\end{array}$ & Maestría \\
\hline $\begin{array}{l}\text { (Universidad de } \\
\text { California en San } \\
\text { Diego, 2017) }\end{array}$ & $\begin{array}{l}\text { Human-Centered } \\
\text { Design: an } \\
\text { Introduction }\end{array}$ & Un mes & $\begin{array}{lr}\begin{array}{l}\text { Metodología } \\
\text { prototipado, } \\
\text { heurística }\end{array} & \text { DCU, } \\
\end{array}$ & Licenciatura \\
\hline $\begin{array}{l}\text { (Universidad de } \\
\text { Minnesota, 2018) }\end{array}$ & $\begin{array}{l}\text { User Interface } \\
\text { Design }\end{array}$ & $\begin{array}{l}\text { Cinco } \\
\text { meses }\end{array}$ & $\begin{array}{l}\text { Factores Humanos en el } \\
\text { diseño del software, } \\
\text { instrumentos para levantar } \\
\text { requisitos, prototipado, } \\
\text { evaluación de usabilidad }\end{array}$ & Licer \\
\hline $\begin{array}{l}\text { (Universidad de } \\
\text { Michigan, 2017) }\end{array}$ & $\begin{array}{l}\text { User } \\
\text { Experience } \\
\text { UX) Research } \\
\text { and Design }\end{array}$ & $\begin{array}{l}\text { Doce } \\
\text { meses }\end{array}$ & $\begin{array}{ll}\text { Factores Humanos en el } & \text { el } \\
\text { diseño del software, } \\
\text { instrumentos para levantar } \\
\text { requisitos, prototipado, } \\
\text { evaluación de usabilidad } \\
\end{array}$ & Licenciatura \\
\hline $\begin{array}{l}\text { (Universidad de } \\
\text { Stanford, 2017) }\end{array}$ & $\begin{array}{l}\text { Design Thinking } \\
\text { for } \\
\text { Experience User } \\
\text { Design, } \\
\text { Prototyping } \quad \& \\
\text { Evaluation } \\
\end{array}$ & $\begin{array}{l}\text { Cuatro } \\
\text { meses }\end{array}$ & $\begin{array}{l}\text { Principios de Diseño, } \\
\text { prototipado y evaluación. }\end{array}$ & Licenciatura \\
\hline $\begin{array}{l}\text { (Universidad de } \\
\text { Uppsala, 2018) }\end{array}$ & $\begin{array}{l}\text { Human- } \\
\text { Computer } \\
\text { Interaction }\end{array}$ & $\begin{array}{l}\text { No lo } \\
\text { especifica }\end{array}$ & $\begin{array}{l}\text { Psicología cognitiva, métodos } \\
\text { de análisis de usuarios, } \\
\text { definición de tareas, pruebas } \\
\text { de usabilidad. }\end{array}$ & Maestría \\
\hline (UNAM, 2016) & $\begin{array}{l}\text { Computación } \\
\text { Gráfica } \\
\text { interacción } \\
\text { humano } \\
\text { computadora } \\
\end{array}$ & $\begin{array}{l}\text { Seis } \\
\text { meses }\end{array}$ & Diseño de interfaces & Licenciatura \\
\hline
\end{tabular}

Tabla 1. Universidades que ofrecen cursos de IHC. Fuente: Elaboración propia.

Para la presente investigación, se ha encontrado como una guía el trabajo de (Valero, 2008) quién a través de su tesis de pregrado denominado Formalización del modelo para el Diseño de la Interacción Humano Computadora (MODIHC) resume los artefactos mínimos a obtenerse para cumplir con el Diseño Centrado al Usuario guiado a través de la Norma ISO 13407, integrando también algunos instrumentos para la recolección de la información.

Sumado a ello, aplicar una estrategia basada en proyectos (ABP) para el seguimiento del DCU sobre un escenario de la vida real, permite vincular al 
estudiante a instruirse en un ambiente de trabajo en equipo, colaborativo y que inclusive le permiten identificar participaciones multidisciplinares para hallar la solución de una problemática, que en el mejor de los casos represente un beneficio para la sociedad.

Son diversos los autores que han aplicado esta estrategia como una solución didáctica durante el seguimiento de un curso escolar (véase Tabla 2). Incluso en (Astorga Vargas, Flores Rios, \& Ibarra Esquer, 2015) se realiza el estudio a detalle del impacto del ABP como estrategia de enseñanza-aprendizaje en diferentes universidades de nuestro país. Sin embargo, su definición e integración dentro de un curso de IHC hasta el momento de esta publicación no ha podido ser identificada, por lo que su inclusión resulta atractiva para mejorar la experiencia de aprendizaje de los estudiantes.

\begin{tabular}{|c|c|c|}
\hline Artículo & Institución & Asignatura \\
\hline $\begin{array}{l}\text { (Hernández, } \\
\text { Heydrich, Rojas, \& } \\
\text { Hernández, 2010) }\end{array}$ & $\begin{array}{l}\text { Universidad de la } \\
\text { Habana }\end{array}$ & Ecología Microbiana \\
\hline (Pérez, 2008a) & $\begin{array}{l}\text { Universidad } \\
\text { Caracas }\end{array}$ & $\begin{array}{ll}\text { Tecnología } & \text { de } \\
\text { Materiales } & \end{array}$ \\
\hline (ITESM, 2005) & $\begin{array}{l}\text { Tecnológico } \\
\text { Monterrey }\end{array}$ & $\begin{array}{l}\text { Ecología Aplicada } \\
\text { Avanzada }\end{array}$ \\
\hline $\begin{array}{lr}\text { (Labra Gayo, } & \text { Gández Lanvin, } \\
\text { Fernández } \\
\text { Calvo Salvador, \& } \\
\text { Cernuda del Río, } \\
\text { 2006) }\end{array}$ & $\begin{array}{l}\text { Universidad } \\
\text { Oviedo }\end{array}$ & $\begin{array}{l}\text { Programación } \\
\text { Declarativa }\end{array}$ \\
\hline (Aliane, 2006) & $\begin{array}{l}\text { Universidad } \\
\text { Europea } \\
\text { Madrid }\end{array}$ & Robótica \\
\hline $\begin{array}{l}\text { (Arellano-pimentel, } \\
\text { Nieva-garcía, \& } \\
\text { Algredo-badillo, 2013) }\end{array}$ & $\begin{array}{l}\text { Universidad del } \\
\text { Istmo }\end{array}$ & Compiladores \\
\hline
\end{tabular}

Tabla 2. Instituciones que se han convertido en casos de éxito por emplear ABP como estrategia de enseñanza-aprendizaje. Fuente: Elaboración propia. 


\section{Marco Teórico}

\subsection{Diseño Centrado en el Usuario bajo la Norma ISO 13407}

(Lorés, Granollers, \& Lana, 2002) define al DCU como una metodología de diseño de software para desarrolladores y diseñadores que emerge de la IHC, ayudando esencialmente a construir aplicaciones que conozcan las necesidades de sus usuarios finales. Por tanto, es una estrategia para gestionar proyectos de creación, mejora e implementación de productos interactivos, de tal manera que se garantice el éxito del sistema resultante. EI DCU no es un proceso genérico independiente del proyecto, sino que se encuentra fuertemente relacionado con los usuarios, las funcionalidades y el contexto (Domingo \& Pera, 2010).

La Norma originalmente nombrada ISO 13407:1999 especifica las recomendaciones necesarias para la inclusión de la metodología del DCU en el ciclo de vida del desarrollo del software interactivo, entendiendo a la interactividad como el proceso para establecer comunicación entre hombre y computadora mediante una interfaz (Usability Net, 2006). Esta norma la cual es objeto de nuestro estudio, define al DCU como "un método iterativo donde se enfatiza involucrar de forma activa al usuario y el entendimiento claro de los requerimientos de la tarea y el usuario" (AENOR, 2000).

Su esquema general puede verse en la Figura 1.

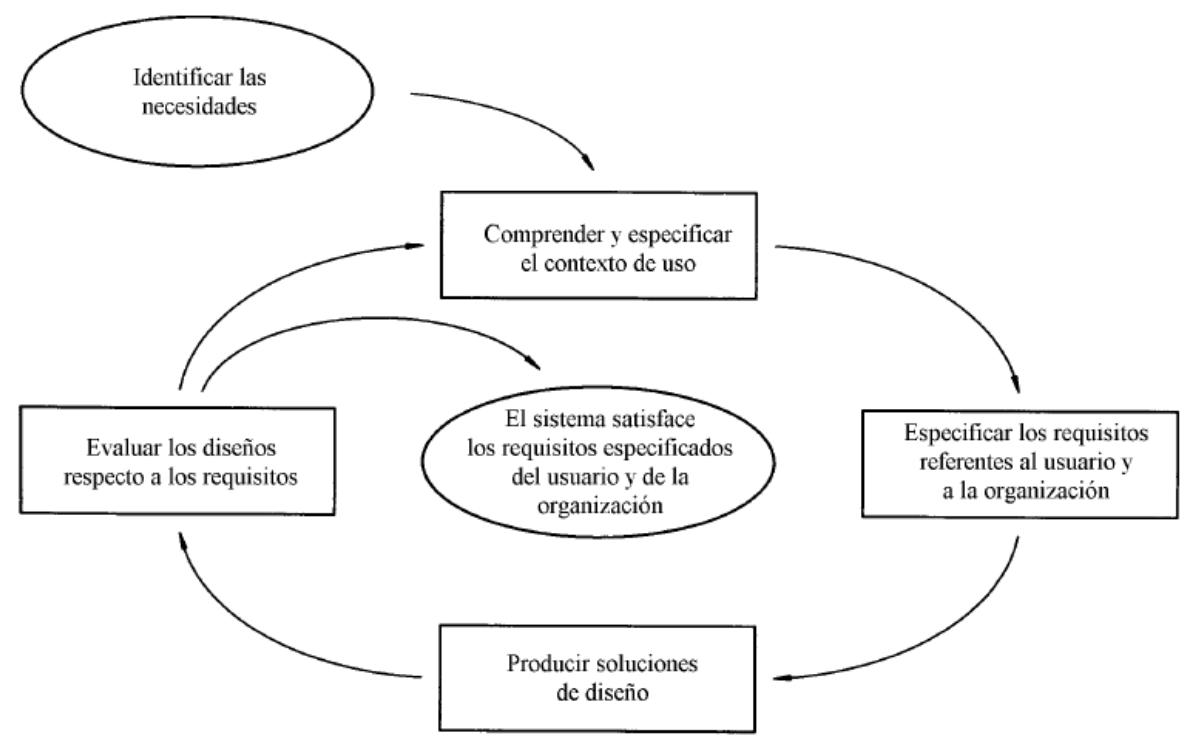

Figura 1. Metodología del Diseño Centrado en el Usuario. Fuente: ISO 13407 (AENOR, 2000) 
La descripción de las etapas que la conforman se establece a continuación:

- ETAPA 1 Comprensión y especificación del contexto de uso: corresponde a la identificación de los usuarios (perfil del usuario), tareas (requerimientos funcionales), y entornos de organización y físicos que definen el contexto en el que se utilizará el sistema (aspectos que pueden reflejarse en el diseño de la interfaz).

- ETAPA 2 Especificación de los requisitos referentes al usuario y a la organización: representa a las características funcionales del sistema, requisitos legislativos, de seguridad, la cooperación y comunicación entre usuarios y su logística propia, reparto de las tareas entre usuarios, el diseño y organización del trabajo, el diseño de la interfaz y la distribución del puesto de trabajo. Es importante que se aclare qué actividades serán propias con ayuda de la tecnología y cuáles no.

- ETAPA 3 Producir soluciones de diseño: etapa en la que se establecen prototipos a partir de la información colectada en los puntos anteriores, la experiencia y los conocimientos de los desarrolladores.

- ETAPA 4 Evaluar los diseños respecto a los requisitos: se determinan los procedimientos de evaluación a emplear que permitirán contribuir a la mejora del diseño de la interfaz, determinar si se han alcanzado los objetivos del usuario y de la organización, etc.

Para una mejor compresión de los productos que conforman la ETAPA 1 se sugiere la revisión de una norma complementaria denominada ISO 9241-11.

\subsection{ISO 9241-11}

La Norma ISO: 9241-11:1998 fue publicada en su versión en español por la Asociación Española de Normalización y Certificación AENOR la cual la nombró como Norma Española UNE-EN-ISO 9241-11, ésta "explica cómo las medidas de los resultados obtenidos por el usuario y su propia satisfacción pueden utilizarse para determinar de qué manera afecta cualquier componente del sistema a su totalidad durante su funcionamiento". Una de sus principales aportaciones radica en su ANEXO A, en cual detalla un formato ejemplo para la definición del contexto de uso, correspondiente a uno de los entregables de la primera etapa del DCU, de igual manera en el ANEXO B se define a través de un ejemplo los indicadores para medir la usabilidad del software desarrollado, útil en la etapa de evaluación (AENOR, 1998). 


\subsection{Aprendizaje basado en proyectos}

Surge del enfoque constructivista de Piaget, Dedwey, Bruner y Vigotsky, el cual sostiene que el estudiante "construye" nuevo conocimiento a partir de conocimientos actuales o previos, por lo que la manera idónea para promover construcciones mentales radica en permitirles a los estudiantes trabajar en escenarios o problemáticas reales y familiares para él, es decir, que representen un valor personal que lo ayude a comprometerse con su solución, permitiendo con ello adquirir aprendizaje significativo (Pérez, 2008a).

(Coria Arreola, 2011) señala algunas ventajas de integrar el ABP dentro de cursos universitarios, entre ellos se encuentran: Promueve que los estudiantes piensen y actúen con base en el diseño de un proyecto, elaborando un plan con estrategias definidas para dar solución a una interrogante, permite el aprender en la diversidad al trabajar en equipo, estimula el crecimiento emocional, intelectual y personal mediante experiencias directas con personas y estudiantes ubicados en diferentes contextos, aprenden a evaluar el trabajo de sus pares, a dar retroalimentación constructiva tanto para ellos mismos como para sus compañeros, alienta a los estudiantes a experimentar al realizar aprendizaje basado en descubrimientos, aprenden de sus errores a enfrentar y superar retos difíciles e inesperados.

En (ITESM, 2005) se sugiere una serie de pasos a seguir cuando se desea plantear un $\mathrm{ABP}$, los cuales consisten en:

a) Antes de la planeación del proyecto: se define la duración, complejidad, tecnología, alcances y personal de apoyo involucrado en el éxito de la dinámica.

b) Metas: representan los objetivos académicos que se espera logren los alumnos al finalizar el proyecto.

c) Resultados esperados en los alumnos: se identifican los objetivos específicos de aprendizaje en base a las metas establecidas.

d) Preguntas guía: conducen al alumno hacia el logro de los objetivos del proyecto.

e) Productos: son construcciones, presentaciones y exhibiciones realizadas durante el proyecto.

f) Actividades de Aprendizaje: llevan a los alumnos a profundizar en los contenidos de conocimientos y a desarrollar actividades de frente a las necesidades del proyecto.

g) Apoyo instruccional: representa a la instrucción y apoyo que permite guiar el aprendizaje de los alumnos y facilitar el desarrollo del proyecto.

h) El ambiente de aprendizaje: se considera establecer las condiciones de trabajo de tal manera que estimule el interés de los alumnos por sus proyectos. 
i) Identificación de recursos: representan a los recursos de información y tecnológicos para que los alumnos logren desarrollar sus proyectos.

Bajo la integración de todas estas vertientes, se describe a continuación la propuesta metodológica empleada.

\section{Propuesta Metodológica}

Dentro de la Universidad del Istmo campus Tehuantepec, en la carrera de Ingeniería en Computación, se imparte durante el séptimo semestre la asignatura denominada "Interacción Humano Computadora" (IHC), la cual tiene como objetivo principal "Proporcionar al estudiante las bases generales para el desarrollo de software centrado en el usuario, mostrando conocimiento en las áreas de usabilidad, desarrollo centrado al usuario y el desarrollo de interfaces." (Universidad del Istmo, 2016) y se encuentra conformado por 7 unidades temáticas: i) Introducción a la IHC, ii) Diseño Centrado en el Usuario, iii) Análisis de requerimientos de usuarios, iv) Diseño conceptual, v) Diseño e implementación, vi) Evaluación de usabilidad, vii) Panorama de investigación en IHC.

Como parte del contenido temático presente en la asignatura de IHC se identifica a la Unidad 2 como la columna vertebral del curso, debido a que es en ésta cuando se presenta la metodología que encausará la dinámica de trabajo durante el semestre, obteniéndose como producto final un software funcional o semifuncional, así, el seguimiento del proyecto deberá corresponder a la integración de los conceptos involucrados en las unidades 2-6 considerando el enfoque de ABP.

\subsection{Alcance del Proyecto}

Para la integración de la dinámica de ABP se decidió trabajar con proyectos a corto plazo, con una complejidad manejable para el estudiante en el transcurso de un semestre, de tal manera que esta dinámica le permita integrar conceptos asociados de la IHC, Ingeniería de Software, Programación, Bases de Datos, Diseño Gráfico, etc., empleando para ello toda la tecnología a su alcance y sujeto a las características propias del problema a resolver; se espera que los proyectos tengan un beneficio a la sociedad, de tal manera que sus usuarios finales sean seleccionados alrededor del contexto en el que viven los alumnos, teniendo finalmente el apoyo del docente adscrito a la asignatura, así como también de colaboradores en diseño gráfico y cómputo para retroalimentar sus avances.

Para este primer esfuerzo se considera la configuración de los productos entregables y los tiempos asociados como una Autonomía limitada por parte del usuario, debido a que el docente requiere llevar un control de los temas vistos y 
solicitar los entregables en tiempos determinados ya que se corre el riesgo de no terminarlos sino se enmarca las actividades desde el inicio.

\subsection{Metas}

El desarrollo semestral de estos proyectos se realizó en el marco de que los estudiantes puedan cumplir las siguientes metas:

- Identificar la relación existente entre los estándares ISO 13406 Y 9241 11.

- Aplicar el DCU a un producto de software que resuelva una necesidad de la vida real.

- Integrar los conceptos atendidos en las unidades temáticas en el producto de software mientras el estudiante demuestra un incremento gradual de sus conocimientos y dominio de los términos durante sus discusiones.

- Identificar y aplicar pruebas de usabilidad con y sin la intervención del usuario.

- Presentar formalmente su solución al cierre del curso mediante una defensa justificada de su solución.

\subsection{Resultados esperados en los alumnos}

A continuación, en la Tabla 3 se describen algunos aspectos considerados como los resultados idóneos que serán adquiridos por los estudiantes durante la aplicación del ABP.

\begin{tabular}{|c|c|}
\hline Conocimiento y desarrollo de habilidades & Resultados del proceso de trabajo \\
\hline $\begin{array}{l}\text { Al finalizar el proyecto, el alumno será capaz de: } \\
\text { - Identificar áreas de oportunidad para } \\
\text { desarrollo de software en que pueda } \\
\text { integrar el DCU como marco de trabajo. } \\
\text { Relacionar e integrar en su léxico } \\
\text { conceptos del área de IHC tales como: } \\
\text { modelo mental, usuario final, perfil del } \\
\text { usuario, metáfora, estilo de interacción, } \\
\text { usabilidad, etc. } \\
\text { - Aplicar estrategias de recolección de } \\
\text { datos en el escenario de estudio para } \\
\text { recabar información que les permita } \\
\text { proponer sus soluciones de diseño. } \\
\text { Aplicar pruebas de usabilidad formales } \\
\text { en función al número de usuarios y las } \\
\text { variables que desee medir. } \\
\text { Desarrollar su capacidad para } \\
\text { interactuar con el grupo de usuarios } \\
\text { seleccionados. }\end{array}$ & $\begin{array}{l}\text { Las competencias y actitudes que los alumnos } \\
\text { aprenderán serán: } \\
\text { - La capacidad de proporcionar críticas } \\
\text { constructivas a los proyectos de sus } \\
\text { compañeros. } \\
\text { Desarrollar una capacidad de } \\
\text { compromiso y profesionalismo para } \\
\text { entregar un software de calidad. } \\
\text { - Un mejor dominio de sus conocimientos } \\
\text { en IHC, así como de su dicción para } \\
\text { justificar la solución de su problemática. } \\
\text { - Desarrollará una mayor disposición } \\
\text { para emprender retos semejantes en } \\
\text { futuras asignaturas. } \\
\text { Descubrirá lo valioso de su labor, así } \\
\text { como su participación activa en un } \\
\text { grupo multidisciplinar. }\end{array}$ \\
\hline
\end{tabular}

Tabla 3. Resultados esperados en los alumnos bajo el ABP. Fuente: Elaboración propia. 


\subsection{Preguntas guía}

A continuación se enlistas las preguntas de referencia que enmarcan el logro de las metas establecidas en el punto 4.2:

1. ¿Qué problemática social identificas en tu localidad que pueda ser resuelta mediante un software interactivo? Considera que deberá ser necesario la presencia de usuarios reales que darían uso a tu solución.

2. ¿Cuáles son los productos relevantes en cada fase del DCU?

3. ¿Qué perfil describiría a tus usuarios finales?

4. ¿Cómo es el contexto de uso del usuario?

5. Bajo observación ¿cómo describirías el modelo mental de tu usuario final?

6. ¿Cuáles son los requerimientos funcionales y no funcionales de tu solución?

7. ¿Cómo organizarás los requerimientos funcionales del software junto al perfil del usuario en un prototipo?

8. Identifica las tareas de usabilidad que emplearás al aplicar alguna de las técnicas de usabilidad vistas en clase (por ejemplo, Thinking Aloud).

\subsection{Productos}

En cada una de las fases del DCU se detallan las actividades que deberán cumplirse dejando a la tarea del alumno-desarrollador los métodos o estrategias a emplear para su realización. Por ejemplo, si bien el DCU señala dentro de la etapa 4 que deben efectuarse procedimientos de evaluación (véase sección 3.1), la norma no establece qué pruebas de usabilidad emplear, dejándolo a criterio del desarrollador en función a coste y tiempo.

Tratando de resolver esta situación y durante una revisión en los trabajos relacionados, se identificó la propuesta de (Valero, 2008) como aporte crucial para la identificación de requisitos elementales durante el seguimiento de las primeras 2 etapas del DCU. Para dar solución a dicho inconveniente, se integraron en un solo esquema de trabajo los productos relevantes que se deben cubrir en cada etapa, fusionando los aportes de la ISO 13407 (AENOR, 2000) y la ISO 9241-11 (norma que sugiere atributos para conformar el perfil del usuario) (AENOR, 1998), junto con la de (Valero, 2008) e integrando conceptos adicionales propios de los contenidos temáticos del curso de IHC. Además, se sugieren estrategias de recolección de información y herramientas de software que agilizan la captación de la información en el esquema de trabajo diseñado para el curso semestral, véase la Tabla 4.

A continuación se describen los entregables de las dos últimas etapas de la metodología por considerarse la principal aportación de esta investigación: 
1. PRUEBA DE USABILIDAD: REVISIÓN POR EXPERTOS: Consiste en una sesión de retroalimentación entre profesores expertos en el área de diseño, de IHC y alumnos para que estos últimos puedan colectar las observaciones pertinentes y con ello mejorar sus propuestas iniciales (prototipos de baja-mediana gama), dicha acción permite reflejar a los miembros participantes en la dinámica como un grupo multidisciplinar, en el que cada rol contribuye en una sección particular de la solución. Como resultado de la dinámica, el alumno obtiene un listado de mejoras futuras que serán trabajadas sobre el prototipo. Mas sin embargo, son los alumnos quienes determinan las mejoras pertinentes a ser tomadas en cuenta.

Una vez efectuada las mejoras, se obtendría una segunda versión del prototipo en mediana gama, que será reportada a criterio del estudiante en su documentación. Posteriormente, se procederá a efectuar:

2. PRUEBA DE USABILIDAD THINKING ALOUD: el estudiante como parte de su aportación documental, describirá la técnica y definirá las tareas de usabilidad que desee probar en su prototipo, para posteriormente seleccionar a 2 usuarios potenciales que cubran el perfil de usuario descrito. Seguido de ello, se aplicará la prueba de usabilidad y se enlistarán los problemas de usabilidad detectados con la clasificación a la que pertenecen (ya sea fallo en la tarea -FT-, pérdida de funcionalidad PF-, problema mayor -PM-, problema menor -PMEN-, molestia -MOL-) ya analizados previamente en clase, reportando sus resultados en un tabulador. La técnica de evaluación Thinking Aloud fue seleccionada para esta actividad ya que es una de las pruebas más sencillas dentro de los métodos que involucran al usuario, así como por su popularidad (Nielsen, 2012) debido a que el atributo que nos interesa medir es la facilidad de aprendizaje de la herramienta en el usuario final y dicha prueba es la adecuada para ello. Su aplicación y la forma de reportar los resultados de la dinámica provienen del autor Soren Lauesen (Lauesen, 2005) por su claridad al efectuarla.

Finalmente, el estudiante reportará la tercera versión de su software en un prototipo de alta gama con las mejoras aplicadas a partir de los problemas de usabilidad detectados. Para homogeneizar el seguimiento del proyecto se procedió a definir un esquema de trabajo a partir de entregables y tabuladores que resumiesen los aspectos principales de cada una de las etapas definidas en el DCU.

El Cierre del curso se verá representado por una ponencia formal por parte de los estudiantes quienes presentarán los aspectos relevantes de su software solución y los resultados de las pruebas de usabilidad empleadas a sus prototipos, obteniendo de ello una retroalimentación inmediata por parte de un 
equipo de profesores pertenecientes a la plantilla de la carrera de Ingeniería en Computación para señalarles las mejoras y los trabajos futuros.

\subsection{Actividades de Aprendizaje}

Como puede comprobarse en la sección 4.5 algunas actividades de aprendizaje irán orientadas a la Planeación de su marco de trabajo en función a la delimitación del problema que se atenderá. Así como también se promoverá la Investigación sobre conceptos y estrategias de recolección de información, posteriormente el estudiante aplicará los conceptos en su proyecto y desarrollará prototipos de su solución que será sometido a pruebas para su mejora continua, finalizando con una presentación formal.

\subsection{Apoyo Instruccional}

A lo largo del desarrollo del curso de IHC se guiará el aprendizaje del grupo mediante sesiones diseñadas para exposición y discusión de temas por parte del docente, así como su intervención con escenarios de ejemplos apoyado de recursos digitales y/o herramientas de su interés. Se eligieron 3 puntos de control relevantes dentro de la dinámica de trabajo, el primero corresponde a la definición de los entregables de la unidad 2 y 3 de la Tabla 4 , el segundo correspondería a los entregables y primer prototipo de las unidades 4, 5 y 6 junto con los resultados de la prueba con expertos, para posteriormente cerrar el proyecto con el tercer punto correspondiente a la versión mejorada del prototipo y los resultados de la prueba Thinking Aloud bajo una presentación formal de resultados.

\subsection{Ambiente de Aprendizaje}

En todo momento se busca promover al estudiante un entorno de motivación para mejorar la calidad de sus productos, a su vez, los proyectos serán diseñados para ser trabajados individualmente o en parejas dependiendo el tamaño del grupo. Durante las sesiones de clase, el grupo se distribuye de tal manera que visualmente tengan acceso a la proyección del material digital y de otros recursos de apoyo a la clase que sean pertinentes para debatir las ideas.

\subsection{Identificación de Recursos}

Desde la primera sesión de clases, los estudiantes tienen acceso a la bibliografía de la asignatura, de igual manera conforme se atienden tópicos se les proporcionan los recursos digitales necesarios para atenderlos en sus proyectos, además del acceso al proyector para sus presentaciones de avances pertenecientes a los puntos de control mencionados en la sección 4.7, cada estudiante hace uso de la tecnología disponible por él. El docente ocasionalmente proporciona plantillas Word prediseñadas que compactan en un 
formato específico los aspectos a cubrir en cada fase del DCU, de tal manera que todos los proyectos presentan un formato de informe homogéneo.

\begin{tabular}{|c|c|c|c|c|}
\hline Etapa del DCU & $\begin{array}{l}\text { Unidad } \\
\text { (es) que } \\
\text { cubre }\end{array}$ & Productos generados & $\begin{array}{l}\text { Estrategias de } \\
\text { recolección y/o } \\
\text { evaluación }\end{array}$ & $\begin{array}{l}\text { Herramientas de } \\
\text { Software para su } \\
\text { realización }\end{array}$ \\
\hline $\begin{array}{l}\text { ETAPA 1: } \\
\text { Comprensión y } \\
\text { especificación del } \\
\text { contexto de uso }\end{array}$ & Unidad 2 & $\begin{array}{l}\text { - Perfil del usuario } \\
\text { - Características de las tareas que los } \\
\text { usuarios deben realizar } \\
\text { - Tabulador con factores a considerar en } \\
\text { el ambiente de trabajo (organizacional, } \\
\text { físico y social.) } \\
\text { - Dispositivos de entrada, salida, } \\
\text { entrada/salida involucrados } \\
\text { - Estilos de interacción a emplear }\end{array}$ & $\begin{array}{l}\text { - Técnica de } \\
\text { observación } \\
\text { - Técnica de } \\
\text { entrevista }\end{array}$ & $\begin{array}{l}\text { Procesadores de texto } \\
\text { bajo el formato de una } \\
\text { plantilla preestablecida: } \\
\text { Word, Open Office, etc. }\end{array}$ \\
\hline $\begin{array}{l}\text { ETAPA 2: } \\
\text { Especificación de } \\
\text { los requisitos } \\
\text { referentes al usuario } \\
\text { y a la organización }\end{array}$ & Unidad 3 & $\begin{array}{l}\text { - Características funcionales del sistema, } \\
\text { reparto de tareas entre usuario y el } \\
\text { sistema }\end{array}$ & $\begin{array}{l}\text { - Técnica de } \\
\text { observación } \\
\text { - Técnica de } \\
\text { entrevista }\end{array}$ & $\begin{array}{l}\text { Procesadores de texto } \\
\text { bajo el formato de una } \\
\text { plantilla preestablecida: } \\
\text { Word, Open Office, etc. }\end{array}$ \\
\hline $\begin{array}{l}\text { ETAPA 3: Producir } \\
\text { soluciones de } \\
\text { diseño }\end{array}$ & $\begin{array}{l}\text { Unidad } 4 \\
\text { y } 5\end{array}$ & $\begin{array}{l}\text { - Software similares } \\
\text { - Metáforas de la interfaz } \\
\text { - Principios de Gestalt } \\
\text { - Prototipo de baja o mediana gama } \\
\text { - Prototipo de mediana gama después de } \\
\text { la 1era. Evaluación (Evaluación por } \\
\text { expertos) } \\
\text { - Prototipo de alta gama después de la } \\
\text { 2da. Evaluación (Thinking Aloud) }\end{array}$ & $\begin{array}{l}\text { - Investigación } \\
\text { de trabajos } \\
\text { relacionados } \\
\text { (software } \\
\text { similares) }\end{array}$ & $\begin{array}{l}\text { - Proto.io (desarrollo de } \\
\text { prototipos de baja- } \\
\text { media gama) } \\
\text { - Gamemaker (proto tipo } \\
\text { de alta gama) } \\
\text { - Photoshop } \\
\text { - Dreamwe aber } \\
\text { - Cualquier entorno de } \\
\text { programación } \\
\text { - Cualquier gestor de } \\
\text { base de datos }\end{array}$ \\
\hline $\begin{array}{l}\text { ETAPA 4: Evaluar } \\
\text { los diseños respecto } \\
\text { a los requisitos }\end{array}$ & Unidad 6 & $\begin{array}{l}\text { - Prueba de revisión con expertos (para } \\
\text { prototipo de baja o mediana gama) } \\
\text { o Lista de observaciones encontradas } \\
\text { - Prueba de usabilidad "Thinking Aloud" } \\
\text { o Descripción de la técnica } \\
\text { o Tareas a evaluar } \\
\text { o Perfil de los usuarios reales a } \\
\text { evaluar } \\
\text { - Gráficos del análisis de resultados } \\
\text { - Tabulador sobre los problemas } \\
\text { detectados y clasificación de éstos } \\
\text { en problemas de usabilidad } \\
\text { o Reporte de mejoras a la interfaz a } \\
\text { partir de los problemas detectados } \\
\text { - Video demostrativo de la aplicación de } \\
\text { la prueba. }\end{array}$ & $\begin{array}{l}\text { - Evaluación } \\
\text { con expertos } \\
\text { - Thinking Aloud }\end{array}$ & $\begin{array}{l}\text { - Herramientas para } \\
\text { tratamiento estadístico } \\
\text { (Excel) } \\
\text { - Aplicaciones para } \\
\text { grabar audio y video } \\
\text { - Procesadores de texto: } \\
\text { Word, Open Office, etc. }\end{array}$ \\
\hline
\end{tabular}

Tabla 4. Propuesta sobre elementos a cubrir, estrategias de recolección y herramientas de software a utilizar en cada etapa del DCU. Fuente: Elaboración propia. 


\section{PUESTA EN PRÁCTICA DE LA ESTRATEGIA $A B P$}

La dinámica consistió en proporcionarles a los estudiantes durante el primer mes del curso los conceptos preliminares concernientes a las Unidades 1 y 2 . Llegado el segundo mes, se solicitó al estudiante una propuesta de su proyecto considerándose título, giro, y justificación, con la intensión de brindarles el asesoramiento que permitiera acotarlos a requerimientos alcanzables en el periodo escolar, así como el de comprender sus propuestas (tal como lo estipula el $A B P)$.

Uno de los proyectos más sobresalientes se denominó Corrector Ortográfico, propuesto por el estudiante Edwin Williams Molina Rodríguez en el ciclo escolar 2014-2015 perteneciente a la carrera de Ingeniería en Computación; el estudiante se inspiró debido a la necesidad de contar con una aplicación móvil que les permitiese a los niños practicar su ortografía, particularmente en el uso de la H, S, C, Z, etc. Para ello, identificó en su entorno a usuarios potenciales y le dio seguimiento bajo la estrategia planteada en la Tabla 4.

Aceptado el proyecto y durante ese mes, además de los temas atendidos en clase, se le fueron solicitando a los estudiantes integrar los conceptos vistos a través de los entregables sugeridos en la Etapa 1 de la Tabla 4, algunas de las cuales se encuentran ejemplificada por el proyecto Corrector Ortográfico en la Figura 2 y Tabla 5. Durante el tercer y cuarto mes, el estudiante recibió la presentación de los temas de las Unidades 3-5, y durante la atención de los temas, ellos procedieron a complementar su proyecto con los entregables correspondientes a las Etapas 2-3 de la Tabla 4, en la Figura 3 se presentan las metáforas aplicadas al proyecto Corrector Ortográfico, las cuales forman parte del diseño de la propuesta de interfaz que resuelve las necesidades de su usuario, así también cumplieron con el desarrollo de un prototipo de baja gama y la identificación de los principios de Gestalt inmersos en sus interfaces (véase la Figura 4a). 


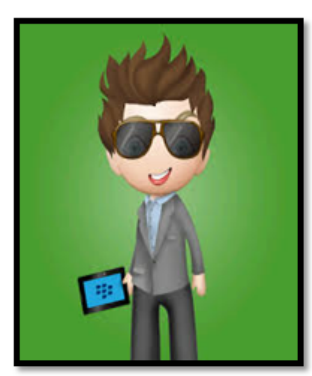

Nombre: Gael Gonzales Molina.

Sexo: Masculino

Edad: 10 años

Lugar de residencia: Salina cruz, Oaxaca

Ocupación: Estudiante de $5^{\circ}$.

Dispositivos favoritos: Celulares, Tablet, Reproductor, tv, Xbox, etc.

Juegos que le gusta: Juegos de plataforma. en una Tablet, con el objetivo de ganar el mayor puntaje posible y competir, basquetbol

Habilidades: inteligente, observador, etc.

Modelo mental: En clase los ponen a escribir un texto, les encierran las palabras con un color rojo, hacen una plana y buscan el significado del mismo, usan libros para leer y comentan entre ellos mismo los errores.

Necesidades: El usuario necesita retroalimentación constante mientras asiste a clases para mejorar su ortografía, además de que lo ayuda al buen desempeño de sus notas, tener más claro lo que lee y aprender más rápido

Actividades: Practica futbol, realiza tareas de investigaciones y recreativas por (medio del internet, revistas, libros), ve la tv, juega con sus amigos, etc.

Figura 2. Representación de la plantilla "Perfil de usuario" para la aplicación “Corrector Ortográfico". Fuente: Alumno Edwin Williams Molina Rodríguez.

Comenzado el quinto mes de trabajo y atendiendo la Unidad 6, se presentó el prototipo de baja gama a un experto en diseño gráfico y al titular de la materia para retroalimentación sobre aspectos a mejorar. Como resultado de la dinámica, el estudiante generó la plantilla correspondiente a la Etapa 4 de la Tabla 4, en la cual incluye las pantallas de su interfaz con las mejoras implementadas, agregando colores a su prototipo y acabados semejantes a una pantalla de un sistema de cómputo o dispositivo móvil; obteniéndose con ello el prototipo de gama media (véase la Figura 4b). Dentro de este caso de estudio, se aplicó el estilo de interacción por manipulación directa. 


\begin{tabular}{|l|l|l|}
\hline Tarea & Características & $\begin{array}{l}\text { Tiempo a realizar } \\
\text { (máximo) }\end{array}$ \\
\hline Instalar el software & $\begin{array}{l}\text { Esta instalación se llevará en cada } \\
\text { dispositivo a utilizar. }\end{array}$ & 1 a 3 min. \\
\hline Crear un usuario & $\begin{array}{l}\text { Con los avances realizados para el } \\
\text { mejor control de su desarrollo. }\end{array}$ & 1 min. \\
\hline $\begin{array}{l}\text { Crear un avatar del } \\
\text { mismo }\end{array}$ & Simular su interacción con el juego & Indefinido \\
\hline $\begin{array}{l}\text { Escoger un sexo } \\
\text { (Multiusuario) }\end{array}$ & $\begin{array}{l}\text { El usurario introducirá el sexo y de } \\
\text { acuerdo al mismo se utilizarán las } \\
\text { herramientas necesarias así como } \\
\text { colores. }\end{array}$ & 30 seg. \\
\hline $\begin{array}{l}\text { Seleccionar tema } \\
\text { dentro del menú }\end{array}$ & $\begin{array}{l}\text { El usuario podrá escoger el nivel o } \\
\text { práctica. }\end{array}$ & 5 seg. \\
\hline Guardar cambios & $\begin{array}{l}\text { El mismo sistema guardara cambios } \\
\text { lapsos de tiempo }\end{array}$ & 3 seg. \\
\hline Realizar los ejercicios & $\begin{array}{l}\text { El usuario tendrá variedad en formas } \\
\text { y gráficos para los repasos. }\end{array}$ & $\begin{array}{l}\text { Dependerá de la } \\
\text { tarea (2-5) min. }\end{array}$ \\
\hline $\begin{array}{l}\text { Obtener recompensas } \\
\text { por los diferentes } \\
\text { ejercicios }\end{array}$ & $\begin{array}{l}\text { El usuario podrá tener recompensas } \\
\text { de las tareas realizadas (dependiendo } \\
\text { el sexo). }\end{array}$ & $\begin{array}{l}\text { Dependerá de la } \\
\text { tarea }\end{array}$ \\
\hline $\begin{array}{l}\text { Calificar al sistema } \\
\text { desplegará una nota mostrando al } \\
\text { usuario la manera de calificar al } \\
\text { sistema con 4 opciones: mala, } \\
\text { regular, buena, excelente }\end{array}$ & 1 min. \\
\hline $\begin{array}{l}\text { Competir con otros } \\
\text { usuarios }\end{array}$ & $\begin{array}{l}\text { El usuario podrá competir con otros } \\
\text { usuarios. } \\
\text { (Desacuerdo a su nivel) }\end{array}$ & $\begin{array}{l}\text { Dentro de 4-6 min. } \\
\text { desempeño realizado }\end{array}$ \\
\hline
\end{tabular}

Tabla 5. Representación de la plantilla "Especificación de tareas funcionales" para la aplicación "Corrector Ortográfico". Fuente: Alumno Edwin Williams Molina Rodríguez.

\begin{tabular}{|c|c|c|}
\hline & Deshacer & $\begin{array}{l}\text { Nuevo } \\
\text { usuario }\end{array}$ \\
\hline & $\begin{array}{l}\text { Respuesta } \\
\text { correcta }\end{array}$ & Ayuda \\
\hline & Continuar & $\begin{array}{l}\text { Jerarquía } \\
\text { de temas y } \\
\text { subtemas }\end{array}$ \\
\hline & Jugar & $\begin{array}{l}\text { Ir a menú } \\
\text { de inicio }\end{array}$ \\
\hline
\end{tabular}

Figura 3. Metáforas de la aplicación "Corrector Ortográfico". Fuente: Alumno Edwin Williams Molina Rodríguez. 


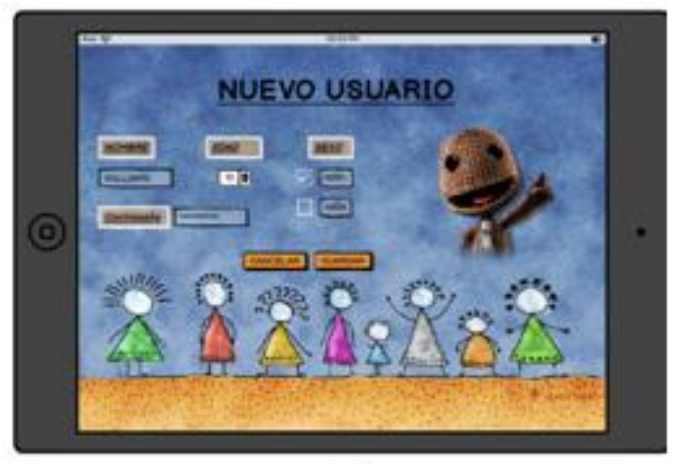

a)

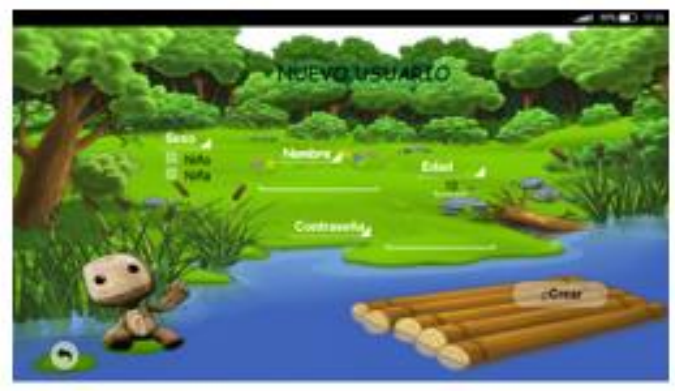

b)

Figura 4. Comparación entre a) prototipo de gama baja contra b) prototipo gama media de la aplicación "Corrector Ortográfico". Fuente: Alumno Edwin Williams Molina Rodríguez.

Durante el mismo mes, el estudiante conoció sobre las pruebas de usabilidad, de tal manera que pudo aplicar la técnica "Thinking Aloud" a su propuesta, con los usuarios reales, dando como resultado la última iteración de la metodología del DCU, reportando con ello un formato semejante al descrito en la Etapa 4 de la Tabla 4 junto a la videograbación de la prueba. Durante el último mes se refinaron los prototipos, para convertirlos en uno de gama alta y se realizó una presentación ante integrantes de la carrera de ingeniería en computación a fin de poder obtener una calificación por la presentación de su trabajo, (véase Figura 5).
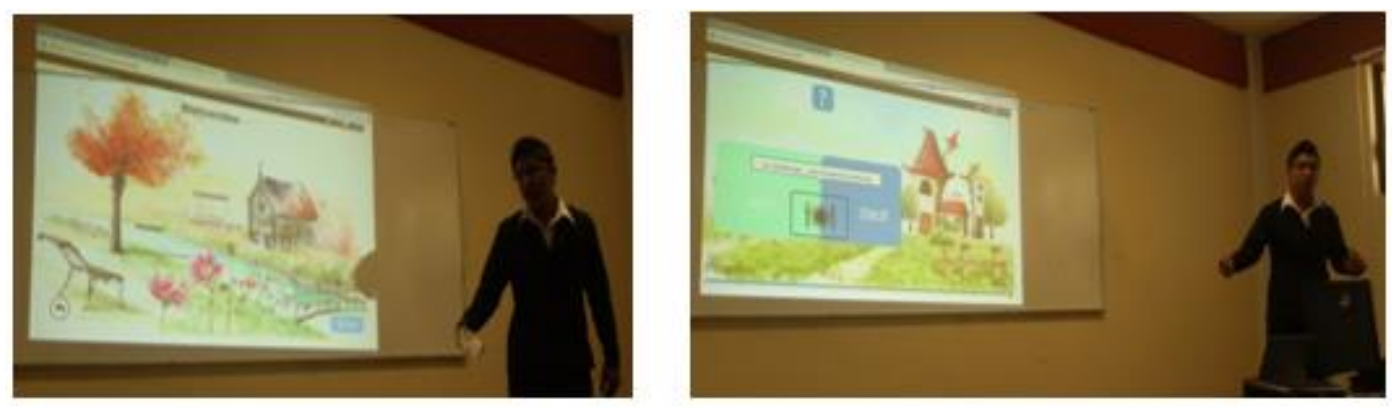

Figura 5. Presentación final de la aplicación "Corrector Ortográfico" ante profesores de la carrera. Fuente: Alumno Edwin Williams Molina Rodríguez. 


\section{Discusión}

Como puede destacarse en la sección anterior, se logró adecuar a los tiempos del semestre las actividades propuesta por la estrategia ABP (ITESM, 2005) tomando en cuenta la distribución de las unidades temáticas del curso de IHC (véase Tabla 4). No obstante, las impresiones de las generaciones de estudiantes partícipes en esta estrategia de trabajo resultan de valiosa aportación para confirmar los aciertos del ABP y realizar mejoras futuras al curso.

Por lo tanto, se procedió a realizar una breve encuesta a estudiantes de las generaciones 2014-2015 y 2016-2017, algunos de ellos ya egresados y otros aún continúan su formación académica. Cabe mencionar que los grupos atendidos en la Universidad del Istmo son reducidos, contemplado una población total de 12 estudiantes entre ambas generaciones, de los cuales solo 10 pudieron establecer contacto para fines de la encuesta.

Con ayuda del instrumento utilizado, se logró colectar impresiones importantes sobre el punto de vista de dos generaciones que dieron uso a la estrategia ABP; entre algunas de las preguntas consultadas se solicitaba al estudiante proporcionar sus impresiones generales sobre el curso, así como las opiniones positivas y negativas encontradas en el trascurso del desarrollo del proyecto, cerrando con sugerencias de mejora y consultando algunos de los conceptos adquiridos en $\mathrm{IHC}$ que hasta ahora siguen aplicando en otras asignaturas o en su vida profesional según fuera su caso; las opiniones fueron congruentes y compartidas, a continuación se enlistan algunas de ellas.

\subsection{Impresiones Generales al Utilizar la Estrategia de Trabajo}

La mayoría de los estudiantes coincidieron en que los alcances de la asignatura eran tan grandes que creían difícil terminarlos y su satisfacción fue grata al percatarse que la metodología de trabajo que los guiaba aceleró la materialización de sus soluciones. A su vez, destacaron la aceptación de tener retroalimentación constante de sus proyectos por parte del docente y la satisfacción obtenida al trabajar con usuarios reales para proponer soluciones innovadoras y apegadas a sus necesidades que les permitieran la mejora de competencias tales como su dicción y redacción.

A continuación se comparte un fragmento proporcionado como respuesta por parte del alumno Carlos Antonio Ortega Vázquez perteneciente a la generación 2016-2017: "La estrategia me pareció adecuada debido a que fue una gran manera de motivación, pues se logró hacer que mis habilidades pudieran desarrollarse, además, las metas puestas fueron alcanzadas y eso me motivó aún más, dado que me demostró que incluso puedo hacer más de lo que podría 
haber creído en un principio, con lo cual adquirí experiencia y empecé a confiar más en mi".

\subsection{Aspectos Negativos Identificados Durante el Desarrollo del Proyecto}

Dentro de las impresiones encontradas por los estudiantes, la mayoría coincidió en sus dificultades para proponer soluciones de diseño, esto debido a que si deseaban incluir animaciones, la manera de plasmar eso dentro de un prototipo de baja gama era muy tedioso; de la misma manera, resultó de dificultad el diseño del instrumento para la recolección de los requisitos del software, esto debido a que nunca habían tenido contacto para tratar con usuarios reales, además de señalar la necesidad de ampliar tiempos para la entrega de los avances del proyecto y su respectivo refinamiento.

Dichas aseveraciones permitieron identificar algunos tiempos desaprovechados en el primer mes del curso escolar en los que se imparte solo teoría para considerar arrancar sus proyectos desde el comienzo del ciclo y así aprovechar al máximo la temporalidad disponible en futuras generaciones.

\subsection{Aspectos Positivos Identificados Durante el Desarrollo del Proyecto}

Los estudiantes demostraron aprobación con la idea de poner en práctica los conocimientos vistos en clase a partir de un proyecto de la vida real que les permitiera tener contacto con los usuarios que realmente le darían uso al software, lo cual se espera al aplicar la metodología ABP (Pérez, 2008), así como también determinaron como asertiva la inclusión de herramientas de software que sirviesen de apoyo para el desarrollo de los prototipos, junto con la dinámica de obtener retroalimentación por parte del usuario final.

Inclusive coincidieron en que el ambiente de trabajo promovió en mucho a aumentar su motivación y compromiso con el proyecto, así como también alimentó un entorno de sana competición entre compañeros que al final concluyó con excelentes resultados. A continuación se destacan al respecto unas breves líneas en la opinión del alumno Emmanuel Marcial Castillo de la generación 2016-2017: "Fue muy bueno que el proyecto se basara en un caso real y que se le fuera dando seguimiento, hasta la presentación final con profesores expertos en diversas áreas (diseño, programación y $\mathrm{HCl}$ )".

Por su parte el alumno Edwin Williams Molina Rodríguez de la generación 20142015 menciona "El alcance del curso fue grande, se adquieren muchos conocimientos tratando de resolver dificultades que se presentan al desarrollar el proyecto como saber el impacto que podía tener la aplicación y conocer 
personas que estuvieran interesadas". Ambas opiniones realzan la ventaja destacada del ABP según (Coria Arreola, 2011) que se enfoca en el desarrollo de nuevas competencias y actitudes de emprendimiento.

En general, la retroalimentación constante de docentes en las áreas afines y usuarios finales representó una atmósfera motivacional que alentó al estudiante a emprender mejoras cada vez más aterrizadas en sus proyectos finales.

\subsection{Sugerencias de Mejora para la Aplicación de la Estrategia en Siguientes Generaciones}

La mayoría de los estudiantes coincidieron en que mostrar proyectos afines de generaciones anteriores para ofrecer un primer alcance e impresión de lo esperado en el curso sería de gran ayuda, ya que contrarrestaría las primeras impresiones mencionadas en la sección 6.1 relacionadas a la imposibilidad de terminar el cometido, además de que mencionan les permitiría comprender el alcance del proyecto y a su vez proponer ideas innovadoras y no repetitivas a las ya realizadas por generaciones anteriores.

También indicaron que si bien el perfil del usuario ayuda a identificar la muestra poblacional a la que se dirige el proyecto solución, consideran de interés explorar la capacidad de intuitividad del software, incluyendo a sus evaluaciones a usuarios que carecieran de tener experiencias con la misma tecnología trabajada, esto para tener un refinamiento de sus prototipos aún más acertadas, no obstante, se es consciente que un curso de IHC por 6 meses es muy breve para poder adecuar todos los intereses de los estudiantes a un curso escolar, sin embargo, esta propuesta daría pie a investigaciones posteriores al curso con la intención de analizar a profundidad la calidad de su software y alentarlos a la escritura de artículos de investigación.

Además, insistieron en la necesidad de compactar el curso escolar a lapsos de teoría más breve para invertir mayor tiempo en el desarrollo de sus proyectos sin que el curso pierda la esencia de la estrategia, lo que actualmente se está intentando refinar.

\subsection{Conceptos De IHC que aún Emplean en su Vida Escolar o Profesional}

Una de las interrogantes más interesantes cuestionadas a este grupo de alumnos y al cual se le dio especial atención, consistió en conocer cuáles eran las nociones del curso que aún conservaban, considerando que un grupo de ellos ya son profesionistas y otros están próximamente por graduarse. 
Las respuestas obtenidas fueron favorecedoras, ya que todos recordaron los conceptos esenciales y enfatizaron la importancia de aplicarlos durante la fase del desarrollo de un software en cualquier necesidad, ya sea académica o profesional, entre los conceptos más mencionados son: definición del perfil del usuario, estándares y reglas de diseño, diseño centrado en el usuario, usabilidad , el diseño iterativo, metáforas, estética (lo visual es muy importante), organización y jerarquías, la documentación del proyecto, adelantarse a posibles errores y prevenir que ocurran, principios de Gestalt.

Todos estos conceptos fueron atendidos con éxito durante las unidades plasmadas en la Tabla 4, muchos de ellos aplicadas implícita o explícitamente en el transcurso de sus proyectos, por lo que resulta satisfactorio saber de la trascendencia de estos conocimientos en estudiantes que han aprendido mediante la estrategia ABP. Además, se logró validar aquellas metas que se deseaban obtener al cierre del curso escolar, tal como se describen en la Tabla 3.

\subsection{Evaluación del Estudiante Respecto a la Estrategia ABP}

Por último, se solicitó a cada estudiante someter a una evaluación en escala del 1-10 su experiencia bajo la estrategia impartida en la clase (ABP) y su satisfacción al cierre de la misma respecto a los proyectos culminados, obteniéndose un promedio de 9.4 y 8.7 respectivamente, lo cual proporciona un margen aceptable para las primeras dos generaciones involucradas y motiva al docente en un proceso de mejora continua de su propuesta de clase. Se espera que en generaciones futuras y aplicadas las sugerencias de mejora proporcionadas por las anteriores generaciones, el promedio de aceptación durante la aplicación de la estrategia mejore y con ello se obtengan más y mejores proyectos innovadores.

\section{Conclusiones}

El presente trabajo desarrolla e implementa una metodología para el seguimiento de un curso de IHC enfocado al DCU enmarcado en una estrategia de ABP. Esta experiencia les permitió a los estudiantes identificar áreas de oportunidad en la vida real que pueden resolverse a través del desarrollo de software, logrando palpar el impacto de la tecnología en sus usuarios finales, que generalmente son familiares y amigos para quienes resuelven una necesidad de información.

La integración del ABP fue aceptado positivamente por los estudiantes quienes en todo momento mostraron una actitud participativa y comprometida con sus proyectos. Esto permitió un aprendizaje acorde a lo esperado ya que los estudiantes participes de la estrategia adquirieron conocimientos elementales 
del enfoque centrado en el usuario para el desarrollo de software de calidad que, a decir de ellos mismos, aún recuerdan y aplican tanto en su vida académica como en su desempeño profesional. Además, un aspecto no menos relevante resultó la participación de expertos en el área de diseño brindándoles la confianza de sentirse inmersos en un grupo multidisciplinar y a su vez garantizar la obtención de un producto de calidad al finalizar las etapas de elaboración.

A su vez, como docente, se logró mapear los conceptos vistos en clase a través de una dinámica atractiva al estudiante con el cual se les consiguió promover el sentido de responsabilidad al tratar de resolver con ayuda computacional los temas escogidos, así como al trabajar y presentar sus avances en estricta formalidad a sus profesores y compañeros de clase.

Finalmente, el proceso de mejora seguido durante la evaluación de los prototipos, busca que los estudiantes puedan proponer artículos de divulgación que resuman su experiencia de aprendizaje y logren con ello obtener un producto de software de calidad para ser sometido a revistas de investigación arbitradas.

\section{Referencias}

AENOR. (1998). Requisitos ergonómicos para trabajos de oficina con pantallas de visualización de datos (PDV) basada en la norma UNE-EN ISO 9241-11.

AENOR. (2000). UNE EN ISO 13407 Procesos de diseño para sistemas interactivos centrados en el operador humano: Junio 2000 (ISO 13407:1999). España: AENOR. Retrieved from https://books.google.com.mx/books?id=L3cjMQAACAAJ

Aliane, N. (2006). Una Experiencia De Aprendizaje Basado En Proyecto En Una Asignatura De Robotica, 01-10.

Arellano-pimentel, J. J., Nieva-garcía, O., \& Algredo-badillo, I. (2013). Aprendizaje Basado en Proyectos Utilizando L-Systems en un Curso de Compiladores. Programación Matemática y Software, 5(1), 81-96.

Astorga Vargas, A. M., Flores Rios, B. L., \& Ibarra Esquer, J. E. (2015). Impacto del aprendizaje basado en proyectos implementado en una empresa escolar de Base Tecnológica dedicada al desarrollo de Software. ReCIBE, 4(4).

Coria Arreola, J. M. (2011). El aprendizaje por proyectos: una metodología diferente. E-FORMADORES, 5(2009), 1-8.

Domingo, M. G., \& Pera, E. M. (2010). Diseño centrado en el usuario. Universitat Oberta de Catalunya. Retrieved from 
https://www.exabyteinformatica.com/uoc/Informatica/Interaccion_persona_o rdenador/Interaccion_persona_ordenador_(Modulo_3).pdf

Hernández, J. A., Heydrich, M., Rojas, M., \& Hernández, A. (2010). Aprendizaje basado en proyectos: una experiencia de innovación docente. Revista Universidad EAFIT, 46(158), 11-21.

ITESM. (2005). Las estrategias y técnicas didácticas en el rediseño.

Labra Gayo, J. E., Fernández Lanvin, D., Calvo Salvador, J., \& Cernuda del Río, A. (2006). Una Experiencia de aprendizaje basado en proyectos utilizando herramientas colaborativas de desarrollo de software libre. XII Jornadas de Enseñanza Universitaria de La Informática.

Lavesen, S. (2005). User interface design: a software engineering perspective. Pearson Education.

Lorés, J., Granollers, T., \& Lana, S. (2002). Introducción a la interacción personaordenador. Universidad de Lleida.

Nielsen, J. (2012). Thinking Aloud: The \# 1 Usability Tool.

Pérez, M. (2008a). Aprendizaje basado en proyectos colaborativos. Una experiencia en educacion superior. Laurus, 14(28), 158-180. Retrieved from http://www.redalyc.org/articulo.oa?id=76111716009

Pérez, M. (2008b). Aprendizaje basado en proyectos colaborativos. Una experiencia en educacion superior. Laurus, 14(28), 158-180.

S. Pressman, R. (2006). Ingeniería del Software Un Enfoque Práctico. Editorial Pais.

UNAM. (2016). Computación Gráfica e Interacción Humano Computadora. Retrieved May 9, 2018, from http://www.ingenieria.unam.mx/programas_academicos/licenciatura/Compu tacion/2016/asignaturas_computacion_2016.pdf

Universidad Autónoma de Barcelona. (2017). Curso de diseño centrado en el usuario. Retrieved January 24, 2018, from http://www.uab.cat/web/postgrado/curso-en-diseno-centrado-en-elusuario/detalle-curso-1206597475768.html/param 1-1793_es/param2-2006/

Universidad de California en San Diego. (2017). Human-Centered Design: an Introduction. Retrieved January 24, 2018, from https://es.coursera.org/learn/human-computer-interaction 
Universidad de Chile. (2017). Curso diseño de experiencias de usuario en sitio web y soportes digitales. Retrieved January 1, 2018, from http://www.uchile.cl/cursos/68077/curso-diseno-de-experiencias-de-usuarioen-sitio-web

Universidad de Michigan. (2017). User Experience (UX) Research and Design. Retrieved May 8, 2018, from https://www.edx.org/micromasters/michiganx-userexperience-ux-research-and-design

Universidad de Minnesota. (2018). User Interface Design. Retrieved May 8, 2018, from https://es.coursera.org/specializations/user-interface-design

Universidad de Stanford. (2017). Design Thinking for User Experience Design, Prototyping \& Evaluation. Retrieved May 9, 2018, from http://hci.stanford.edu/courses/cs147/2017/au/

Universidad de Uppsala. (2018). Human-Computer Interaction. Retrieved May 9 , 2018, from http://www.uu.se/en/admissions/master/selma/Kurser/?kKod=1MD016\&typ=1\&i $d=$

Universidad del Istmo. (2016). Programa de Estudios Interacción Humano Computadora. Sto. Dgo. Tehuantepec, Oaxaca: Universidad del Istmo. Retrieved from http://www.unistmo.edu.mx/ computacion/otros/temarios/2016/7_SEMESTRE/3 072 Interacción Humano-Computadora.pdf

Usability Net. (2006). ISO 13407 Human centred design processes for interactive systems. Retrieved February 4, 2018, from http://www.usabilitynet.org/tools/13407stds.htm

Valero, G. (2008). Formalización del Modelo para el Diseño de la Interacción Humano-Computadora (MODIHC). Universidad de los Andes.

\section{Notas biográficas:}

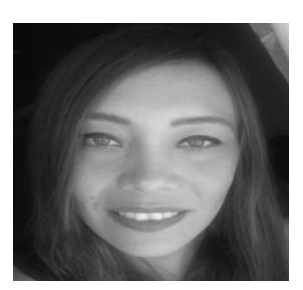

Guadalupe Toledo Toledo es Ingeniera en Sistemas Computacionales por el Instituto Tecnológico de Tuxtepec, y Maestra en Computación Aplicada por parte del Laboratorio Nacional de Informática Avanzada. Actualmente es ProfesorInvestigador de tiempo completo adscrito a la carrera de Ingeniería en Computación en la Universidad del Istmo. Sus áreas de interés se centran en el desarrollo de software y prototipos didácticos con aplicación en ingeniería y computación, aplicación de evaluaciones de 
usabilidad a productos de software centrados en el usuario y la integración del cómputo aplicado en la solución de problemas multidisciplinares.

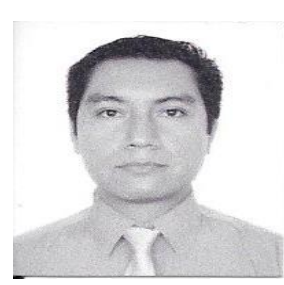

J. Jesús Arellano Pimentel es Ingeniero en Sistemas Computacionales por el Instituto Tecnológico de Morelia en 2002 y Maestro en Ciencias en Ingeniería Eléctrica con opción en Sistemas Computacionales por la Universidad Michoacana de San Nicolás de Hidalgo en 2005. Actualmente se desempeña como Profesor-Investigador de Tiempo Completo adscrito a la carrera de Ingeniería en Computación en la Universidad del Istmo campus Tehuantepec, Oaxaca, México. Sus áreas de interés incluyen: software educativo, realidad virtual, prototipos didácticos, compiladores, y robótica móvil.

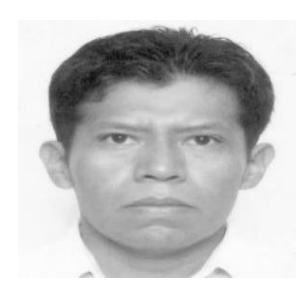

Francisco Aguilar Acevedo es Ingeniero en Electrónica por la Universidad Tecnológica de la Mixteca y Maestro en Ciencias en Ingeniería Mecatrónica por el Centro Nacional de Investigación y Desarrollo Tecnológico. Actualmente es Profesor-Investigador de tiempo completo adscrito a la carrera de Ingeniería en Computación en la Universidad del Istmo, y miembro activo del cuerpo académico de Cómputo Aplicación. Sus áreas de interés incluyen: la inteligencia artificial, la robótica, los sistemas embebidos y la instrumentación electrónica.

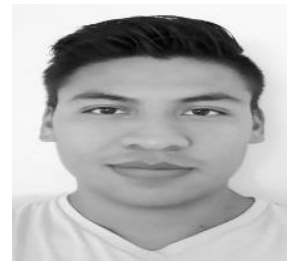

Edwin Williams Molina Rodríguez es estudiante de la generación 2013-2018 de la carrera de Ingeniería en Computación en la Universidad del Istmo. Actualmente trabaja en un proyecto de investigación para desarrollar un prototipo capaz de generar energía a base de hidrógeno para la industria automotriz. Sus áreas de interés se encuentran orientadas hacia las tendencias tecnológicas en beneficio del planeta y el área de desarrollo humano.

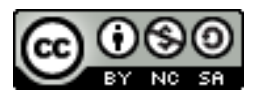

Esta obra está bajo una licencia de Creative Commons Reconocimiento-NoComercial-Compartirlgual 2.5 México. 